\title{
Opacity and effective field theory in anti-de Sitter backgrounds
}

\author{
Sylvain Fichet \\ Walter Burke Institute for Theoretical Physics, California Institute of Technology, \\ Pasadena, California 91125, USA \\ and ICTP South American Institute for Fundamental Research and IFT-UNESP, \\ R. Dr. Bento Teobaldo Ferraz 271, São Paulo, Brazil
}

(Received 5 September 2019; published 4 November 2019)

\begin{abstract}
We consider quantum field theory in a five-dimensional anti-de Sitter background, possibly truncated by four-dimensional (4D) branes. In the Euclidian version of this space, it is known that propagators exponentially decay when they go far enough toward the Poincaré horizon, i.e. deep enough into the IR region of the AdS bulk. In this note, we show that an analogous property exists in Lorentzian AdS. The exponential suppression is found to occur in the presence of bulk interactions dressing the AdS propagators. We calculate one-loop gravitational dressing and find that the suppression effect comes from the scalar component of the 5D graviton. We then argue that, at least at strong coupling, this exponential decay censors the region of spacetime where the 5D effective field theory would become invalid. As an application we estimate the rate for the cascade decay of bulk fields-which are known to produce soft spherical events with high multiplicity - and find this rate to be exponentially suppressed.
\end{abstract}

DOI: $10.1103 /$ PhysRevD.100.095002

\section{INTRODUCTION}

Quantum field theory in anti de-Sitter spacetime has been a source of deep formal insights as well as elegant phenomenological developments for at least two decades. Formally, investigations of the AdS/CFT conjecture and its holographic realization have brought knowledge about the theories on both sides of the correspondence [1-3]. Phenomenologically, AdS space and its truncated or deformed versions have offered a rich playground for physics beyond the Standard Model, providing for instance a simple explanation to the electroweak hierarchy problem [4].

In five dimensions, the field theory living in the $\mathrm{AdS}$ background should be seen as an effective field theory (EFT) valid up to some cutoff on short five-dimensional (5D) distances $\left|\Delta X^{M}\right| \sim 1 / \Lambda$, beyond which the whole 5D model is superseded by a theory of quantum gravity. When described in the Poincare patch with conformal coordinates $X^{M}=\left(x^{\mu}, z\right)$, a striking difference between AdS space and flat space is that the validity limit of the EFT is also reached for small enough 4D distance $\left|x^{\mu}\right|$ in such a way that the validity cutoff of the EFT depends on the position in the bulk of AdS. Namely, the validity cutoff of the theory scales

\footnotetext{
*sylvain.fichet@gmail.com
}

Published by the American Physical Society under the terms of the Creative Commons Attribution 4.0 International license. Further distribution of this work must maintain attribution to the author(s) and the published article's title, journal citation, and DOI. Funded by SCOAP ${ }^{3}$. along the fifth dimension as $1 / z$. This essential fact as long been known (see, e.g., [4,5]), and it is perhaps in [6] that it has been made clear that the position-dependent cutoff simply results from the presence of higher dimensional operators in the effective 5D theory.

Another feature inherent to curved space, known in the Euclidian version of AdS, is that propagators become exponentially suppressed when one of the end points in the fifth dimension goes too far in the IR. In positionmomentum space, if a propagator carries a Euclidian absolute four-momentum $p$, the exponential suppression occurs beyond $p \sim 1 / z$ [6]. Interestingly, this region matches roughly the region in which the 5D EFT would break down. Therefore, in Euclidian AdS, it turns out that propagators refuse to go in the region where the EFT is invalid: in a sense the theory censors itself.

However, Euclidian AdS is of limited interest since it does not contain particles. A more interesting question is whether the propagators have a similar behavior in Lorentzian AdS. Since the position-dependent cutoff is also present in Lorentzian AdS, one may wonder if a similar mechanism of "censorship" of the IR region may happen, which would require a decay of the propagator in the IR as in the Euclidian case.

This question is the main focus of the present note: we study how and to what extent the propagators decay in Lorentzian AdS. This is a feature of relevance both for the sake of thoroughly understanding effective field theory in AdS and in view of subsequent developments for physics beyond the Standard Model such as a "warped 
dark sector" [7]. The steps taken in the paper are as follows. In Sec. II, we lay down the AdS setup needed for our analysis. The behavior of the scalar propagator in the different regions of position-momentum space is studied in Sec. III. We then turn our attention to the effect of the imaginary part of the 1PI insertions dressing the propagator. After introducing a Kaluza-Klein sum trick in Sec. IV, we investigate the simple case of dressing by a cubic scalar interaction in Sec. V. The dressing of the propagator by $5 \mathrm{D}$ gravity is then evaluated in Sec. VI. Comments about censorship of the EFT are made in Sec. VII A. As a corollary to our study, and taking profit of the formalism, we study the rate of cascade decay of bulk fields in Sec. VIII. Section IX contains our conclusions, and details on the graviton loop calculation are given in the appendix.

\section{A SLICE OF ADS}

The full action used in this paper describes a scalar field and gravity in a slice of $\operatorname{AdS}_{5}$. The action takes the form

$$
S_{\mathrm{AdS}}=\int d^{5} X^{M} \sqrt{|g|}\left[M_{*}^{3}(\mathcal{R}-\Lambda)+\mathcal{L}_{\Phi}\right],
$$

where spacetime is taken to be $\mathrm{AdS}_{5}$ with cosmological constant $\Lambda=-12 k^{2}, k$ being the AdS curvature. The $M_{*}$ parameter sets the strength of $5 \mathrm{D}$ gravity. ${ }^{1} \mathcal{R}$ is the Ricci scalar, and $\mathcal{L}_{\Phi}$ describes a $5 \mathrm{D}$ scalar field. The graviton Lagrangian is expanded in Sec. VI. In this section, our focus is instead on the scalar field. The metric of the AdS background is denoted by $\gamma_{M N}$, such that $g_{M N}=\gamma_{M N}+\cdots$ where the ellipse denotes the metric fluctuations.

We use the Poincaré patch with conformal coordinates

$$
d s^{2}=\gamma_{M N} d X^{M} d X^{N}=(k z)^{-2}\left(\eta_{\mu \nu} x^{\mu} x^{\nu}-d z^{2}\right),
$$

where $\eta_{\mu \nu}$ is Minkowski metric with $(+,-,-,-)$ signature. The values $z=0, z \rightarrow \infty$ correspond, respectively, to the AdS boundary and to the Poincare horizon. The fifth dimension is assumed to be compact with $z \in\left[z_{0}, z_{1}\right]$, where $z_{0} \equiv 1 / k, z_{1} \equiv 1 / \mu$ are, respectively, referred to as UV and IR branes.

The Lagrangian for the scalar reads

$$
\mathcal{L}_{\Phi}=\frac{1}{2} \nabla_{M} \Phi \nabla^{M} \Phi-\frac{1}{2} m_{\Phi}^{2} \Phi^{2}+\mathcal{L}_{\mathrm{int}}+\mathcal{L}_{\mathcal{B}}
$$

where $m_{\Phi}^{2}$ is the scalar bulk mass, $\mathcal{L}_{\text {int }}$ describes bulk matter interactions, and $\mathcal{L}_{\mathcal{B}}$ describes $4 \mathrm{D}$ brane-localized

\footnotetext{
${ }^{1}$ With this convention, we have the matching $M_{*}^{3} \approx k M_{\mathrm{Pl}}^{2}$ in the 4D low-energy theory.
}

Lagrangians. The scalar bulk mass has to satisfy the Breitenlohner-Freedman bound $m_{\Phi}^{2} \geq-4 k^{2}$ to prevent tachyonic instabilities in $\mathrm{AdS}_{5}$ [8].

Extremizing the action in $\Phi$ gives the classical 5D equation of motion

$$
\mathcal{D} \Phi=0,
$$

with the operator

$$
\mathcal{D}=\frac{1}{\sqrt{\gamma}} \partial_{M}\left(\gamma^{M N} \sqrt{\gamma} \partial_{N}\right)+m_{\Phi}^{2}
$$

and two boundary conditions

$$
\left.\mathcal{B}^{\mathrm{UV}} \Phi\right|_{z_{0}}=0,\left.\quad \mathcal{B}^{\mathrm{IR}} \Phi\right|_{z_{1}}=0,
$$

where the $\mathcal{B}^{\mathrm{UV}, \mathrm{IR}}$ operators depend on the brane localized Lagrangians. The Feynman propagator $\Delta\left(X, X^{\prime}\right)$ of the free scalar in the AdS background is the Green function satisfying the equation of motion

$$
\mathcal{D}_{X} \Delta\left(X, X^{\prime}\right)=-i \frac{\delta^{(5)}\left(X-X^{\prime}\right)}{\sqrt{\gamma}},
$$

as well as the boundary conditions, Eq. (6).

From now on we work in momentum space along the 4D Minkowski directions such that the coordinates are $\left(p^{\mu}, z\right)$. The Greek indexes are understood to be contracted using the $\eta_{\mu \nu}$ metric. We have $\partial_{\mu} \partial^{\mu} \Phi=-p_{\mu} p^{\mu} \Phi$. On introduces $p=\sqrt{p_{\mu} p^{\mu}}$, which is real (imaginary) for timelike (spacelike) four-momentum $p^{\mu}$. The solutions to the equation of motion Eq. (4) in the 5D position-momentum space are given by

$$
z^{2} J_{\alpha}(p z), \quad z^{2} Y_{\alpha}(p z)
$$

where the parameter $\alpha \in \mathbf{R}$ is related to the scalar bulk mass by $\alpha^{2}=\frac{m_{\Phi}^{2}}{k^{2}}+4$. The boundary operators applied to the solutions Eq. (8) are denoted as ${ }^{2}$

$$
\begin{aligned}
& \mathcal{B}^{\mathrm{UV}, \mathrm{IR}}\left[z^{2} J_{\alpha}(p z)\right]_{z_{0}, z_{1}} \equiv \tilde{J}_{\alpha}^{\mathrm{UV}, \mathrm{IR}}, \\
& \mathcal{B}^{\mathrm{UV}, \mathrm{IR}}\left[z^{2} Y_{\alpha}(p z)\right]_{z_{0}, z_{1}} \equiv \tilde{Y}_{\alpha}^{\mathrm{UV}, \mathrm{IR}} .
\end{aligned}
$$

With these definitions, the Feynman propagator is

\footnotetext{
${ }^{2}$ The overall factors of $\tilde{J}_{\alpha}^{\mathrm{UV}}, \tilde{J}_{\alpha}^{\mathrm{IR}}$ are arbitrary, since the boundary operators are defined up to a constant. These overall constants cancel inside the expression of the propagator.
} 


$$
\begin{aligned}
\left\langle\Phi(p, z) \Phi\left(-p, z^{\prime}\right)\right\rangle \equiv & \Delta_{p}\left(z, z^{\prime}\right)= \\
& i \frac{\pi k^{3}\left(z z^{\prime}\right)^{2}}{2} \frac{\left[\tilde{Y}_{\alpha}^{\mathrm{UV}} J_{\alpha}\left(p z_{<}\right)-\tilde{J}_{\alpha}^{\mathrm{UV}} Y_{\alpha}\left(p z_{<}\right)\right]\left[\tilde{Y}_{\alpha}^{\mathrm{IR}} J_{\alpha}\left(p z_{>}\right)-\tilde{J}_{\alpha}^{\mathrm{IR}} Y_{\alpha}\left(p z_{>}\right)\right]}{\tilde{J}_{\alpha}^{\mathrm{UV}} \tilde{Y}_{\alpha}^{\mathrm{IR}}-\tilde{Y}_{\alpha}^{\mathrm{UV}} \tilde{J}_{\alpha}^{\mathrm{IR}}},
\end{aligned}
$$

where $z_{<}=\min \left(z, z^{\prime}\right), z_{>}=\max \left(z, z^{\prime}\right)$.

Finally, let us specify the brane terms. For our purposes, it is enough to consider

$$
\mathcal{L}_{\mathcal{B}}=\sqrt{\bar{\gamma}} \frac{1}{2} \Phi^{2}\left(\delta\left(z-z_{0}\right) M_{\mathrm{UV}}-\delta\left(z-z_{1}\right) M_{\mathrm{IR}}\right),
$$

where $\bar{\gamma}_{\mu \nu}$ is the induced metric on the branes, giving $\sqrt{\bar{\gamma}}=(k z)^{-4}$. It is convenient to express the brane masses as

$$
M_{\mathrm{UV}}=(\alpha-2) k-b_{\mathrm{UV}} k, \quad M_{\mathrm{IR}}=(\alpha-2) k+b_{\mathrm{IR}} k .
$$

The boundary conditions [Eq. (6)] then give

$$
\begin{aligned}
\tilde{J}_{\alpha}^{\mathrm{UV}} & =\frac{p}{k} J_{\alpha-1}\left(\frac{p}{k}\right)-b_{\mathrm{UV}} J_{\alpha}\left(\frac{p}{k}\right), \\
\tilde{J}_{\alpha}^{\mathrm{IR}} & =\frac{p}{\mu} J_{\alpha-1}\left(\frac{p}{\mu}\right)+b_{\mathrm{IR}} J_{\alpha}\left(\frac{p}{\mu}\right)
\end{aligned}
$$

and similarly for the $Y_{\alpha}$ functions.

In these conventions, a massless mode is present in the spectrum when $b_{i}=0$ or $b_{i}=2 \alpha$. Note this becomes manifest when $b_{\mathrm{UV}}=b_{\mathrm{IR}}=0, \alpha=2$ or $b_{\mathrm{UV}}=-b_{\mathrm{IR}}=-2 \alpha$, $\alpha=-2$, since in both cases one has $m_{\Phi}=0, M_{i}=0$ so that all mass terms vanish in the 5D Lagrangian. The sign of the $b_{i}$ 's is chosen such that positive $b_{i}$ means a positive mass contribution to the $4 \mathrm{D}$ modes.

\section{LIMITS OF THE PROPAGATOR}

Here we study the behavior of the propagator $\Delta_{p}\left(z, z^{\prime}\right)$ in the various regions of position-momentum space. We can notice that the change $\left(b_{\mathrm{UV}} \rightarrow b_{\mathrm{UV}}+2 \alpha, b_{\mathrm{IR}} \rightarrow b_{\mathrm{IR}}-2 \alpha\right)$ reverses the sign of $\alpha$ in the boundary conditions. A subsequent change $\alpha \rightarrow-\alpha$ gives back the original boundary condition, and we can check that the full propagator remains unchanged under these operations. Hence, there is a symmetry in the solutions and for our purpose it is convenient to focus on $\alpha \geq 0$. The $b_{\mathrm{UV}}, b_{\mathrm{IR}}$ parameters do not play an important role for this work and will remain unspecified.

For $p$ real (i.e. $p^{\mu}$ timelike) and larger than $\mu$, the propagator has an infinite series of Kaluza Klein (KK) poles with $O(\mu)$ spacing, whose masses are given by the equation

$$
\tilde{J}_{\alpha}^{\mathrm{UV}} \tilde{Y}_{\alpha}^{\mathrm{IR}}-\tilde{Y}_{\alpha}^{\mathrm{UV}} \tilde{J}_{\alpha}^{\mathrm{IR}}=0
$$

The propagator can be written as an infinite sum over the KK modes, but this representation is not of immediate use; our focus here is rather on the closed form of the propagator.

We first consider the low-momentum region $|p| \lesssim \mu$ (region 1), where we have

$$
\Delta_{p}\left(z, z^{\prime}\right) \approx i \frac{k^{3}\left(z z^{\prime}\right)^{2-\alpha}\left(2 \alpha-b_{\mathrm{IR}}+b_{\mathrm{IR}}\left(T z_{>}\right)^{2 \alpha}\right)\left(2 \alpha+b_{\mathrm{UV}}-b_{\mathrm{UV}}\left(k z_{<}\right)^{2 \alpha}\right)}{2 \alpha\left(b_{\mathrm{UV}}\left(2 \alpha-b_{\mathrm{IR}}\right) k^{2 \alpha}+b_{\mathrm{IR}}\left(2 \alpha+b_{\mathrm{UV}}\right) T^{2 \alpha}\right)} .
$$

We can see that this term is constant with respect to $p$ and represents a contact operator induced by the heavy KK modes. The expression diverges at $b_{\mathrm{UV}, \mathrm{IR}}=0$ or $b_{\mathrm{UV}}=-b_{\mathrm{IR}}=-2 \alpha$, signaling that a massless mode is also present in these particular cases.
Increasing the momentum, we have the region $\mu \lesssim|p| \lesssim$ $\frac{1}{z_{>}}$(region 2), in which the KK poles along the real axis appear. Let us study the form of the propagator away from the poles,

$$
\begin{aligned}
\Delta_{p}\left(z, z^{\prime}\right) \approx & i \frac{(k z)^{2}\left(k z^{\prime}\right)^{2}}{2 \alpha b_{\mathrm{UV}} k} \frac{1}{\left(z_{>} k\right)^{\alpha}}\left(\frac{b_{\mathrm{UV}}+2 \alpha}{\left(z_{<} k\right)^{\alpha}}-b_{\mathrm{UV}}\left(z_{<} k\right)^{\alpha}\right) \\
& -i \frac{\Gamma(-\alpha)(k z)^{2}\left(k z^{\prime}\right)^{2}}{\Gamma(\alpha+1) 2 b_{\mathrm{UV}}^{2} k}\left(\frac{b_{\mathrm{UV}}+2 \alpha}{\left(z_{>} k\right)^{\alpha}}-b_{\mathrm{UV}}\left(z_{>} k\right)^{\alpha}\right)\left(\frac{b_{\mathrm{UV}}+2 \alpha}{\left(z_{<} k\right)^{\alpha}}-b_{\mathrm{UV}}\left(z_{<} k\right)^{\alpha}\right)\left(\frac{-p^{2}}{4 k^{2}}\right)^{\alpha} .
\end{aligned}
$$


It turns out that, away from the poles, the expression becomes independent of $\mu$ and $b_{\mathrm{IR}}{ }^{3}{ }^{3}$ This signals the fact that the existence of the IR brane is irrelevant away from the poles. The IR boundary condition, unlike the UV one, has no impact on the propagator in this regime, and the IR brane can be sent to infinity $(1 / \mu \rightarrow \infty)$ without affecting Eq. (17).

The first term in Eq. (17) has almost the same form as in the low energy limit except that $b_{\mathrm{IR}}$ is set to zero. From this, we conclude that this term somehow encodes the effect from the heavy modes. In contrast, the second term is nonanalytic in $p$ and one may thus suspect that it encodes the collective effect of light modes. This is easily verified by, for instance, evaluating the nonrelativistic potential induced by the $t$-channel exchange of the $\Phi$ continuum between static sources placed at points $z, z^{\prime}$ in the bulk, and with three-dimensional separation $r=|\mathbf{r}|$. When evaluating the spatial potential, the first term of Eq. (17) is analytic and contribute as a delta function $\delta(r)$, while the second, nonanalytic term induces a nonlocal contribution

$$
V(r) \propto \frac{1}{r} \frac{1}{(k r)^{2+2 \alpha}} .
$$

Since a potential with finite range must result from the exchange of light degrees of freedom, we conclude that the nonanalytic term of Eq. (17) captures the collective contribution of light KK modes. ${ }^{4}$

Increasing again the four-momentum, we then enter the region $\frac{1}{z_{>}} \lesssim|p| \lesssim \frac{1}{z_{<}}$(region 3 ) where the propagator takes the form

$$
\begin{aligned}
\Delta_{q}\left(z, z^{\prime}\right)= & -i \sqrt{\pi}\left(k z_{<}\right)^{2}\left(k z_{>}\right)^{3 / 2} \\
& \times \frac{b_{\mathrm{UV}}\left(k z_{<}\right)^{\alpha}-\left(b_{\mathrm{UV}}+2 \alpha\right)\left(k z_{<}\right)^{-\alpha}}{2 b_{\mathrm{UV}} k \Gamma(\alpha+1)}\left(\frac{p}{2 k}\right)^{\alpha-1 / 2} \\
& \times \frac{\cos \left(\frac{p}{\mu}-p z_{>}\right)}{\cos \left(\frac{p}{\mu}+\frac{\pi}{4}(1-2 \alpha)\right)} .
\end{aligned}
$$

This expression is nonanalytic, but more importantly we can see that whenever $p$ has an imaginary part the whole expression is exponentially suppressed as

$$
\Delta_{p}\left(z, z^{\prime}\right) \propto e^{-|\operatorname{Im} p| z_{>}} .
$$

\footnotetext{
${ }^{3}$ To be slightly more precise, along the real axis and away from the poles the second term is proportional to $S_{\alpha}=\frac{\sin \left(\frac{p}{\mu}-\frac{\pi}{4}(1-2 \alpha)\right)}{\sin \left(\frac{p}{\mu}-\frac{\pi}{4}(1+2 \alpha)\right)}$, which has periodic zeros. But whenever $p$ gets an imaginary part larger than $\sim \pi \alpha \mu$, one gets $S_{\alpha} \approx(-1)^{\alpha}$, which has been used in Eq. (17).

${ }^{4}$ One may notice that in the context of AdS/CFT it is again the nonanalytic term which plays the key role. Namely, in the UV-toUV brane propagator $\Delta_{p}\left(z_{0}, z_{0}\right)$, this term encodes the CFT operator probed by the $\Phi\left(z_{0}\right)$ source. In contrast, the first term of Eq. (17) only gives a mass to the source.
}

For spacelike four-momentum, where $p$ is purely imaginary, the exponential suppression Eq. (46) occurs just like in Euclidian AdS [9]. Instead, for timelike momentum, the propagator is merely oscillating and no exponential suppression occurs. However, we may suspect that the situation changes in the presence of interactions, which dress the propagator with 1PI insertions and induce an imaginary component. A suppression of the kind of Eq. (46) is nevertheless not guaranteed. Hence, a full calculation is required for knowing the behavior of the dressed propagator, which will be carried out in Secs. V and VI. This behavior has no equivalent in flat space.

Finally, at even higher momentum, $\frac{1}{z_{<}} \lesssim|p|$ (region 4), we have

$$
\begin{aligned}
\Delta_{p}\left(z, z^{\prime}\right)= & i \frac{(k z)^{3 / 2}\left(k z^{\prime}\right)^{3 / 2}}{p} \\
& \times \frac{\cos \left(p\left(\frac{1}{T}-z_{>}\right)\right) \cos \left(p z_{<}-\frac{\pi}{4}(1+2 \alpha)\right)}{\sin \left(\frac{p}{T}-\frac{\pi}{4}(1+2 \alpha)\right)} .
\end{aligned}
$$

As well known [5], this propagator resembles very much the flat space propagator in a 5D compact dimension of radius $1 / T$, up to the constant phases and to the nontrivial $(z z)^{3 / 2}$ factor. Away from the real axis, this propagator is exponentially suppressed as

$$
\Delta_{p}\left(z, z^{\prime}\right) \propto e^{-|\operatorname{Im} p|\left|z-z^{\prime}\right|} .
$$

\section{ADS MODE SUMS}

It can be often useful, at least at the conceptual level, to express the propagator $\Delta_{p}\left(z, z^{\prime}\right)$ in a spectral representation, writing it as the infinite sum of KK modes

$$
\Delta_{p}\left(z, z^{\prime}\right)=i \sum_{n=0}^{\infty} \frac{f_{n}(z) f_{n}\left(z^{\prime}\right)}{p^{2}-m_{n}^{2}}
$$

where the $f_{n}(z)$ are the orthonormal KK wave functions. The KK decomposition provides a convenient 4D viewpoint on the 5D physics. ${ }^{5}$ However, in the amplitudes, unless one is interested in one or a few $\mathrm{KK}$ modes, one ends up with sums involving the KK masses $m_{n}$ as well as the profiles $f_{n}(z)$, whose evaluation can be quite challenging. Here, we develop a systematic trick to evaluate the KK sums.

Sums over KK modes appear either in amplitudes with virtual KK modes or in square amplitudes with KK modes in the external legs. Our trick applies to both cases. The general idea is the following. Let us consider a generic quantity

\footnotetext{
${ }^{5}$ An important conceptual pitfall is that the EFT for KK modes breaks down in the IR region; see Sec. VII A.
} 


$$
X=\int d z \int d z^{\prime} \sum_{n=0}^{\tilde{n}} f_{n}(z) f_{n}\left(z^{\prime}\right) A\left(m_{n}^{2} ; z, z^{\prime}\right),
$$

which describes either an amplitude with KK modes in an internal line or a square amplitudes with KK modes in an external line. We can rigorously re-express $X$ using the propagator and a contour integral enclosing the poles up to $\tilde{n}$,

$X=-\frac{1}{2 \pi} \int d z \int d z^{\prime} \oint_{\mathcal{C}[\tilde{n}]} d \rho \Delta_{\sqrt{\rho}}\left(z, z^{\prime}\right) A\left(\rho ; z, z^{\prime}\right)$.

The contour $\mathcal{C}[\tilde{n}]$ is taken counterclockwise. Clearly, putting Eq. (23) in Eq. (25) and using the residue theorem gives back Eq. (24). The interest of Eq. (25) is that we can now use the closed-form expression of the propagator Eq. (16), or its various limits described in Sec. III.

As a simple example, one can consider the creation of real $\mathrm{KK}$ modes from an interaction localized on the UV brane. The coupling of a given KK mode $n$ to the UV brane is proportional to $f_{n}\left(z_{0}\right)$, and the emission rate for the individual mode takes the form

$$
\Gamma_{n}=A\left(m_{n}\right)\left(f_{n}\left(z_{0}\right)\right)^{2},
$$

where $A\left(m_{n}\right)$ depends on $m_{n}$ at least via a phase space factor. The emission of any KK mode is given by

$$
\Gamma=\sum_{n=0}^{\tilde{n}} \Gamma_{n}=\sum_{n=0}^{\tilde{n}} A\left(m_{n}\right)\left(f_{n}\left(z_{0}\right)\right)^{2},
$$

where the heavier mode $\tilde{n}$ is set by the kinematic threshold encoded in $A\left(m_{n}\right)$. The contour integral trick then gives

$$
\Gamma=-\frac{1}{2 \pi} \oint_{\mathcal{C}[\tilde{n}]} d \rho \Delta_{\sqrt{\rho}}\left(z_{0}, z_{0}\right) A(\sqrt{\rho}) .
$$

By the optical theorem, this quantity gives also the imaginary part of the diagram with an internal $\Phi$ line starting and ending on the UV brane. Both quantities are represented in Fig. 1.

Let us briefly turn to a calculation in AdS. A sum that we will be of use in many of the subsequent calculations is

$$
\sum_{n=0}^{\tilde{n}} f_{n}(z) f_{n}\left(z^{\prime}\right)=-\frac{1}{2 \pi} \oint_{\mathcal{C}[\tilde{n}]} d \rho \Delta_{\sqrt{\rho}}\left(z, z^{\prime}\right),
$$

with the $\tilde{n}$ pole lying in region 2 of position-momentum space. In the corresponding limit Eq. (17), the first term is analytic and does not contribute to the integral. The nonanalytic term in Eq. (17) does contribute, because it has a branch cut. In the $\rho$ variable, the branch cut is along the real axis, and the contour $\mathcal{C}[\tilde{n}]$ must cross it since it has to enclose the poles, thereby picking the discontinuity

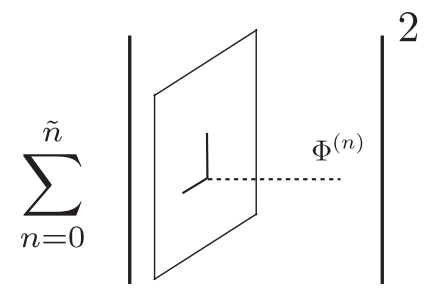

(i)

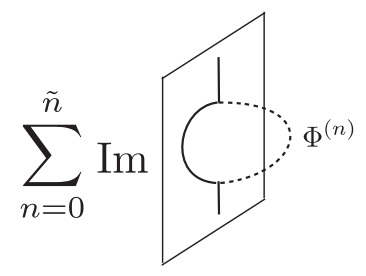

(ii)
FIG. 1. Two typical processes involving KK sums. (i) Emission of KK modes. (ii) Virtual KK modes exchange.

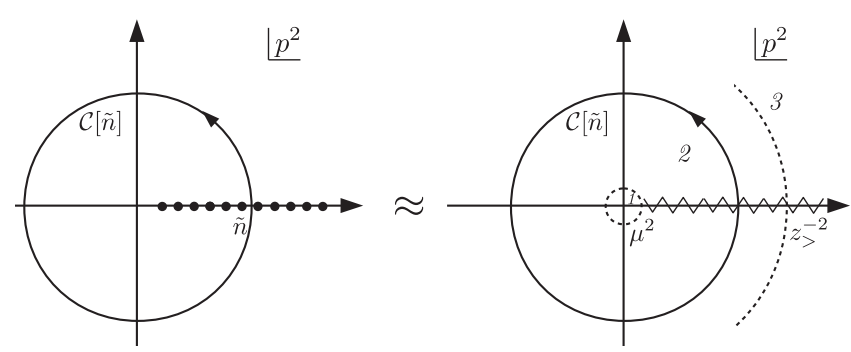

FIG. 2. Contour integration in the $p^{2}$ complex plane. The infinite series of poles from the $\mathrm{KK}$ representation of the propagator is shown on the left-hand side, and the branch cut from its closed form is shown on the right-hand side. The approximation regions are also pictured.

across the real axis. The integration contour and the properties of the propagators are shown in Fig. 2.

Choosing the $\mathcal{C}[\tilde{n}]$ contour to be a circle with radius $A$ enclosing the poles up to $\tilde{n}$, i.e. $\left.\left.\mathcal{C}[\tilde{n}]=\left(A e^{i \theta} \mid \theta \in\right]-2 \pi, 0\right]\right)$ with $m_{\tilde{n}}^{2}<A<m_{\tilde{n}+1}^{2}$, the integral is found to $\mathrm{be}^{6}$

$$
\begin{aligned}
& \sum_{n=0}^{\tilde{n}} f_{n}(z) f_{n}\left(z^{\prime}\right) \\
& \approx \frac{2 k(k z)^{2}\left(k z^{\prime}\right)^{2}}{\Gamma(\alpha+1) \Gamma(\alpha+2) b_{\mathrm{UV}}^{2}}\left(\frac{b_{\mathrm{UV}}+2 \alpha}{\left(z_{>} k\right)^{\alpha}}-b_{\mathrm{UV}}\left(z_{>} k\right)^{\alpha}\right) \\
& \times\left(\frac{b_{\mathrm{UV}}+2 \beta}{\left(z_{<} k\right)^{\alpha}}-b_{\mathrm{UV}}\left(z_{<} k\right)^{\alpha}\right)\left(\frac{m_{\tilde{n}}}{2 k}\right)^{2 \alpha+2} .
\end{aligned}
$$

This expression is approximate since one has used the limit Eq. (17) of the propagator. Also, one has approximated the radius to $m_{\tilde{n}}$ because the spacing between modes is $O(\mu)$ and is negligible at our level of approximation, implying that the KK modes are essentially treated as a continuum. The result of Eq. (30) can be numerically compared to the exact expression, and one finds that the agreement remains of $O(1)$ up to $m_{\tilde{n}}$ of order of a few $1 / z_{>}$, beyond which one

\footnotetext{
${ }^{6}$ The choice of domain of $\theta$ has to do with conventions for Bessel functions, but one can simply check that it is this domain which gives a physical result.
} 
enters too much in region 3 where the approximation breaks down.

This integral is very useful because it often appears when phase space factors are neglected. It can for instance be applied to the case of KK mode emission discussed above, when the KK modes are away from the kinematic threshold. Conversely, these examples are another way to see that the nonanalytic term of Eq. (17) is the one encoding the light component of the $\mathrm{KK}$ continuum. Indeed, it is clear that only light modes can appear in the processes of Fig. 1 because of the kinematic threshold. It turns out that it is the nonanalytic term which contributes to these processes, while the contribution from the analytic term vanishes.

\section{DRESSED PROPAGATOR}

In this section and the next one, we investigate the scalar propagator dressed by one-loop 1PI insertions. ${ }^{7}$ In full generality, given a 1PI diagram in position-momentum space $i \Pi_{p}\left(z, z^{\prime}\right)$, the dressed propagator satisfies

$\mathcal{D} \Delta_{p}\left(z, z^{\prime}\right)-\frac{1}{\sqrt{\gamma}} \int d u \Pi_{p}(z, u) \Delta_{p}\left(u, z^{\prime}\right)=-i \frac{\delta\left(z-z^{\prime}\right)}{\sqrt{\gamma}}$.

One can for instance verify that this is equivalent to the geometric series representation by treating the insertion perturbatively. Writing $\Delta_{p}\left(z, z^{\prime}\right)=\Delta_{p}^{(0)}\left(z, z^{\prime}\right)+$ $\Delta_{p}^{(1)}\left(z, z^{\prime}\right)+\cdots$, we can note that the equation of motion $\Delta_{p}^{(1)}\left(z, z^{\prime}\right)$ is sourced by $\int d u \Pi(z, u) \Delta_{p}^{(0)}\left(u, z^{\prime}\right)$, thus implying

$\Delta_{p}^{(1)}\left(z, z^{\prime}\right)=i \int d u \int d v \Delta_{p}^{(0)}(z, u) \Pi_{p}(u, v) \Delta_{p}^{(0)}\left(v, z^{\prime}\right)$,

which is precisely the term with one $i \Pi$ insertion in the geometric series. ${ }^{8}$ Higher terms of the series are obtained recursively.

As a warm-up in this section, we consider the dressing of $\Phi$ by a loop of another scalar $\varphi$ with cubic bulk interaction

$$
\mathcal{L}_{\text {int }} \supset \lambda \Phi \varphi^{2}
$$

where $\lambda=O(\sqrt{k})$. The bulk mass parameter for $\varphi$ is denoted $\beta$, with $\beta^{2}=\frac{m_{p}^{2}}{k^{2}}+4$. This self-energy diagram is shown in Eq. (34). In terms of Kaluza Klein modes, the diagram involves two sums since there are two internal lines of $\varphi$. For our purposes, we are only interested in the imaginary part of $\Pi$. This means that the KK sums end at the kinematic threshold for production of real KK particles, $p=m_{n}+m_{n^{\prime}}$. Applying the contour integral trick shown in Sec. IV, the imaginary part of the diagram is found to be

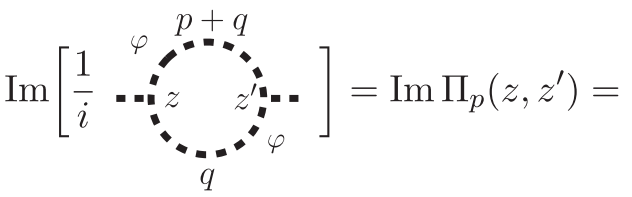

$$
\begin{aligned}
& \frac{1}{8 \pi^{2}} \frac{\lambda^{2}}{(k z)^{5}\left(k z^{\prime}\right)^{5}} \oint_{\mathcal{C}[p]} d \rho \oint_{\mathcal{C}[p-\sqrt{\rho}]} d \rho^{\prime} \Delta_{\sqrt{\rho}}\left(z, z^{\prime}\right) \Delta_{\sqrt{\rho^{\prime}}}\left(z, z^{\prime}\right) \operatorname{Im}\left[\frac{1}{i} \int \frac{d q^{4}}{(2 \pi)^{4}} \frac{1}{\left(p^{2}-\rho\right)\left((p+q)^{2}-\rho^{\prime}\right)}\right] .
\end{aligned}
$$

Our aim is to study the effect of this insertion on the behavior of the propagator. More precisely our focus is on region 3 (i.e. $1 / z_{>} \lesssim|p| \lesssim 1 / z_{<}$). Since in this regime we have $p \gtrsim \frac{1}{z_{>}}$, the values of $\sqrt{\rho}, \sqrt{\rho^{\prime}}$ can lie anywhere with respect to the inverse coordinates of the self-energy, i.e. all the regions of position-momentum space from 1 to 4 are in principle accessible to the propagators inside the loop. However, there are no contributions from propagators lying in region 1 since they are analytic in this region.

\footnotetext{
${ }^{7}$ Exact loop calculations in AdS are typically difficult; see, e.g., $[10,11]$. Our approach relies instead on approximations, including the limits of Sec. III.

${ }^{8}$ For the last step, recall that $\mathcal{D} \Phi=J$ is solved by $\Phi(z)=$ $i \int d u \Delta(z, u) J(u)$.
}

Contributions from regions 2 to 4 are nonanalytic and are thus in principle nonzero.

To proceed in a convenient way, we hypothesize that propagators are actually suppressed in the region $p \gtrsim 1 / z_{>}$. This hypothesis will be checked at the end of the calculation. When assuming it is true, it implies that the propagators in the loop are suppressed for $\sqrt{\rho}, \sqrt{\rho}^{\prime} \gtrsim 1 / z_{>}$, as a result of their own dressing. This implies that the KK sum is truncated at $\sim 1 / z_{>}$, i.e. the contour integral is over a circle of radius $\sim 1 / z_{>}$. It follows that the contributions to the selfenergy come from propagators lying in region 2 , while contributions from regions 3 to 4 are exponentially suppressed. Following this approach, if the hypothesis of suppression for $p \gtrsim 1 / z_{>}$is verified, we will only need to calculate the contribution from the nonanalytic part of the 
propagator in region 2. For concreteness, we take the integration contour with a radius $|\rho|=1 / z_{>}^{2}$; this contour is noted $\mathcal{C}\left[z_{>}^{-1}\right]$.

The imaginary part of the bracket in Eq. (34) is a standard 4D result. The imaginary part of the self-energy is found to be

$$
\begin{aligned}
\operatorname{Im}_{p}\left(z, z^{\prime}\right) \approx & \frac{1}{128 \pi^{3}} \frac{\lambda^{2}}{(k z)^{5}\left(k z^{\prime}\right)^{5}} \oint_{\mathcal{C}\left[z_{>}^{-1}\right]} d \rho \\
& \times \oint_{\mathcal{C}\left[z_{>}^{-1}\right]} d \rho^{\prime} \Delta_{\sqrt{\rho}}\left(z, z^{\prime}\right) \Delta \sqrt{\rho^{\prime}}\left(z, z^{\prime}\right) \frac{K\left(p^{2} ; \rho, \rho^{\prime}\right)}{p^{2}},
\end{aligned}
$$

where

$K\left(p^{2} ; \rho, \rho^{\prime}\right)=\frac{\sqrt{\left(p^{2}-\left(\sqrt{\rho}+\sqrt{\rho^{\prime}}\right)^{2}\right)\left(p^{2}-\left(\sqrt{\rho}-\sqrt{\rho^{\prime}}\right)^{2}\right)}}{p^{2}}$

is the familiar two-body kinematic factor. Since we are assuming we are in the $p>1 / z_{>}$region, the values of $\sqrt{\rho}$, $\sqrt{\rho}$ are small with respect to $|p|$ and we can safely take $K \approx K\left(p^{2} ; 0,0\right)=1$. The contour integrals now reduce to the one already calculated in Sec. IV; see Eqs. (29) and (30). For end points away from the UV brane, the dependence on $b_{\mathrm{UV}}$ disappears [this can be seen in Eq. (17)]. We show only this case for simplicity, the general case works similarly. The final result is found to be

$\operatorname{Im} \Pi_{p}\left(z, z^{\prime}\right) \approx \frac{1}{8 \pi} \frac{\lambda^{2} k^{2}}{\Gamma^{2}(\beta+1) \Gamma^{2}(\beta+2)} \frac{1}{(k z)^{3}\left(k z^{\prime}\right)^{3}}\left(\frac{z_{<}}{4 z_{>}}\right)^{2 \beta+2}$.

At that point we have a simple expression for the 1PI insertion. However, it is still nonlocal and it is thus difficult to solve the dressed equation of motion. To go further, we shall use a position space version of the narrow width approximation (NWA). The position space NWA amounts to a $\partial_{5}$ expansion of $\Pi$ where the $\partial_{5}$ derivatives act on the propagator. ${ }^{9}$ It can be equivalently seen as an expansion over the basis of the Dirac delta's derivatives

$$
\begin{aligned}
\Pi_{p}\left(z, z^{\prime}\right)= & F_{0}(z) \delta\left(z-z^{\prime}\right)-F_{1}(z) \delta^{\prime}\left(z-z^{\prime}\right) \\
& +\frac{F_{2}(z)}{2} \delta^{\prime \prime}\left(z-z^{\prime}\right)+\cdots
\end{aligned}
$$

This can be directly obtained from the dressed equation of motion Eq. (31), where $\Pi_{p}$ is convoluted with $\Delta_{p}$.

\footnotetext{
${ }^{9}$ This 5D NWA should not be confused with a 4D NWA for individual KK modes, which often does not hold for heavy KK modes.
}

The coefficients of the expansion of the $\Pi_{p}$ distribution in Dirac delta's derivative are found to be given by the moments of the distribution, ${ }^{10}$

$$
F_{n}(z)=\int d z^{\prime}\left(z-z^{\prime}\right)^{n} \Pi_{p}\left(z, z^{\prime}\right)
$$

With the NWA expansion Eq. (38), $\Pi_{p}\left(z, z^{\prime}\right)$ is decomposed as a sum of local operators and the equation of motion simplifies to an ordinary differential equation. This is a simplification in itself; however, this trick is truly appealing because of the property of dressing: the local terms can be consistently included step by step in the equation of motion, provided one determines the dressed propagator at every step. For instance, instead of solving in the presence of the two first terms, one can solve for the first term, then dress the obtained solution with the second term. For our purposes, we will not need to go further than the quadratic order.

The three first coefficients $F_{n}(z)$ are given by

$$
\begin{aligned}
& F_{0}(z)=\lambda^{2} k C_{0} \frac{1}{(k z)^{5}}, \quad F_{1}(z)=\lambda^{2} C_{1} \frac{1}{(k z)^{4}}, \\
& F_{2}(z)=\frac{\lambda^{2}}{k} C_{2} \frac{1}{(k z)^{3}},
\end{aligned}
$$

with

$$
\begin{aligned}
\left(\begin{array}{l}
C_{0} \\
C_{1} \\
C_{2}
\end{array}\right)= & \frac{1}{128 \pi} \frac{1}{16^{\beta} \Gamma^{2}(\beta+1) \Gamma^{2}(\beta+2)} \\
& \times\left(\begin{array}{c}
\frac{1}{2 \beta}+\frac{1}{2 \beta+4} \\
\frac{1}{2 \beta+1}+\frac{1}{2 \beta+3}-\frac{1}{2 \beta}-\frac{1}{2 \beta+4} \\
\frac{2}{2 \beta+2}+\frac{1}{2 \beta}+\frac{1}{2 \beta+4}-\frac{2}{2 \beta+1}-\frac{2}{2 \beta+3}
\end{array}\right) .
\end{aligned}
$$

Let us investigate how these local operators deform the free solutions [given by Eq. (8)]. For our purposes, it is enough to consider the effect of each of them separately. The first term of the expansion $\Pi\left(z, z^{\prime}\right) \supset F_{0}(z) \delta\left(z-z^{\prime}\right)$, when included in the dressed equation of motion Eq. (31), gives a contribution whose $z$-dependence is the same as the bulk mass term. Therefore, at leading order in the NWA, the bulk mass develops a (negative) imaginary part. This is similar to what happens to a propagator in flat space. Defining

$$
\tilde{\alpha}=\sqrt{\alpha^{2}-i C_{0} \lambda^{2} / k}
$$

\footnotetext{
${ }^{10}$ This can be guessed by direct computation, or can be found by thinking in terms of the characteristic function of $\Pi_{p}\left(z, z^{\prime}\right)$ in Fourier space.
} 
with $\alpha$ real, the solutions to the homogeneous equation of motion become

$$
z^{2} J_{\tilde{\alpha}}(p z), \quad z^{2} Y_{\tilde{\alpha}}(p z)
$$

instead of the free solution Eq. (8). Having a slightly complex order in the Bessel functions implies that the poles are slightly shifted away from the real axis by a constant amount. That the bulk mass develops an imaginary part because of dressing is certainly a noteworthy feature and is after all what we expect from a 5D theory. However, this feature is not very relevant for our purpose, because the behavior of the propagator is not critically modified and in particular no suppression happens when the propagator lies in region 3 or 4 .

The second term of the expansion $\Pi\left(z, z^{\prime}\right) \supset F_{1}(z) \delta^{\prime}\left(z-z^{\prime}\right)$ induces a $\partial_{5}$ derivative and has the right $z$ factor to change the coefficient of the $\partial_{5}$ term in the $\mathcal{D}$ operator. In the presence of this term, the solutions are deformed to

$$
z^{\gamma} J \sqrt{m_{\Phi}^{2}+\gamma^{2}}(p z), \quad z^{\gamma} Y \sqrt{m_{\Phi}^{2}+\gamma^{2}}(p z),
$$

where $\gamma=2-i C_{1} \lambda^{2} / 2 k$. We can see that this deformation gives again a complex contribution to the order of the Bessel function. It also induces a $z$-dependent phase. But again, these deformations to the free solution are small effects which are not too relevant for our study.

The third insertion $\Pi\left(z, z^{\prime}\right) \supset F_{2}(z) \delta^{\prime \prime}\left(z-z^{\prime}\right)$ modifies the $\partial_{5}^{2}$ term of the $\mathcal{D}$ operator and gives solutions where, in top of a complex Bessel function order and z-dependent phase, the argument of the Bessel function is also changed. Showing only this last effect for simplicity, the solutions take the form

$$
z^{2} J_{\alpha}\left(\frac{p z}{\sqrt{1+i C_{2} \lambda^{2} / 2 k}}\right), \quad z^{2} Y_{\alpha}\left(\frac{p z}{\sqrt{1+i C_{2} \lambda^{2} / 2 k}}\right) .
$$

This deformation is the important one, because it changes the phase of the Bessel's function argument. As a consequence, there are no poles along the real axis, and the Bessel functions rather have an exponential behavior controlled by the imaginary part of the argument. The full propagator in the presence of this deformation is given by the free propagator Eq. (11) where $p$ is replaced by $p / \sqrt{1+i C_{2} \lambda^{2} / 2 k} \approx p\left(1-i C_{2} \lambda^{2} / 4 k\right)$. In the $p \gtrsim 1 / z_{>}$ region, for timelike momentum, the propagator behaves therefore as

$$
\Delta_{p}\left(z, z^{\prime}\right) \propto e^{-C_{2} \lambda^{2} / 4 k p z_{>}} .
$$

From this result, we conclude that bulk interactions induce an exponential suppression of the propagator: the IR region of the Lorentzian AdS background is opaque. This is a quantum effect, unlike the case of spacelike momentum where suppression occurs in the free propagator, the suppression is here controlled by the interaction-dependent, loop-induced parameter $C_{2} \lambda^{2} / 4 k$.

A number of points requires discussion to ensure that our result is solid.

First, regarding the NWA expansion, the higher-order terms do not have a qualitative impact on the exponential behavior found in Eq. (46), precisely because the propagator is already exponentially suppressed in the region of interest. Concretely, insertions with higher powers of $\partial_{5}$ acting on Eq. (46) bring powers of $\left(C_{2} \lambda^{2} / 4 k\right) p z$ but do not change the exponential suppression, which is thus expected to be a robust feature. ${ }^{11}$

Second, let us come back to the hypothesis that the propagators in the loop are suppressed outside of region 2 , such that only region 2 contributes to the loop integral. Since we indeed find exponential suppression, the hypothesis is validated. This basic calculation is sufficient for conceptual discussion, but if one wishes to be more precise, one should take into account that we have truncated the mode sums at $p \approx 1 / z_{>}$[see Eq. (34)], while the suppression found in Eq. (46) occurs more precisely at $p \sim\left(4 k / C_{2} \lambda^{2}\right) / z_{>}$. The truncation of the loop should match this value for the calculation to be more accurate. This is a self-consistent problem which can be solved by iterating the calculation until reaching a fixed point. For instance, a self-consistent result assuming 5D strong coupling $[12,13]$ is

$$
\lambda=24^{1 / 3} \pi \sqrt{k}, \quad \beta=1, \quad z_{>} \lesssim \frac{2.8}{p},
$$

where the last inequality describes the truncation of the loop integral.

Notice that this is at the limit of validity of region 2 . For smaller $\lambda$ or larger $\beta, C_{2}$ gets smaller and there should be KK modes contributing beyond region 2 . The contributions from regions 3 to 4 are however more difficult to evaluate analytically. Going in that direction, one may as well compute the dressed propagators numerically for given $p, z, z^{\prime}$. This is a heavy task which is beyond the scope of this work; a mere estimate is enough for the present study. Our estimate uses only region 2 and is thus conservative since it selects at worse a subset of the KK modes running in the loop.

Third, one may wonder about the magnitude of higherorder loops contributions. The relative magnitude of oneloop and higher-order loop diagrams depends on $p$ and $z$.

\footnotetext{
${ }^{11}$ We may also note that the coefficients of the expansion have a mild hierarchy, with, for instance, $C_{2} / 2 C_{0} \sim 1 / 13$ for $\beta=1$, $C_{2} / 2 C_{0} \sim 1 / 60$ for $\beta=3$, which somewhat reduces the higher terms.
} 
Namely, the higher-order contributions tend to become non-negligible in the IR, at some point inside the $p z_{>}>1$ region. This is expected, since we are in an effective theory with nonrenormalizable interactions. As a matter of fact, one approach to determine the validity of an EFT is precisely to compare loops of different order-the EFT cutoff being then the region of position-momentum space at which loops of different order become of same magnitude. Generally, because of loop suppression, one expects the higher-order loops to become relevant when the exponential suppression from one-loop is already established, such that no crucial qualitative change is expected from higherloop diagrams.

Let us for instance focus on a two-loop self-energy diagram, proportional to $\lambda^{4}$. The imaginary part of the two-loop diagram is tied to the three-body decay calculated in Sec. VIII by the optical theorem. An evaluation in the $p<1 / z_{>}$regime is given in Eq. (84). It turns out that even for the point Eq. (47) the two-loop contribution is suppressed by 2 orders of magnitude with respect to the one-loop contribution. This implies that $p$ has to be sensibly larger than $1 / z_{>}$for the two-loop contribution to become sizable, and the exponential suppression from one-loop is thus already established when this happens.

This concludes our calculation of dressing. The emphasis in this section has been on the conceptual steps and on the approximations taken, considering the dressing from a simple scalar loop. In the next section, we apply the same approach to the gravity case, the steps followed are essentially the same but the calculations are slightly more technical.

\section{GRAVITATIONAL DRESSING}

Five-dimensional gravity is always present in the theory, hence $5 \mathrm{D}$ gravitons induce a universal contribution to the dressing of matter fields. The gravity formalism is somewhat heavier than the scalar case because each KK graviton has 5 degrees of freedom, two of helicity-two, two of helicity-one, one of helicity-zero, each with their own coupling to the stress-energy tensor. The trace of the metric is a nonphysical, ghosty degree of freedom which also contributes to the loop. We closely follow the formalism of [14]. ${ }^{12}$

The canonically normalized metric fluctuation around the AdS background is defined by

$$
g_{M N}=\gamma_{M N}+\sqrt{\frac{2}{M_{*}^{3}}} h_{M N} .
$$

The expansion of the gravity action Eq. (1) up to quadratic order is well known (see, e.g., $[15,16]$ ), giving the action for the 5D graviton $h_{M N}$,

\footnotetext{
${ }^{12}$ To the exception that we use the opposite metric signature.
}

$$
\begin{aligned}
S^{h}=\int & d X^{M} \sqrt{\gamma}\left(\frac{1}{2} \nabla_{R} h_{M N} \nabla^{R} h^{M N}-\frac{1}{2} \nabla_{R} h \nabla^{R} h\right. \\
& +\nabla_{M} h^{M N} \nabla_{N} h-\nabla_{M} h^{M N} \nabla^{R} h_{R N} \\
& \left.+\frac{1}{2}\left(h_{M N}^{2}+h^{2}\right)+\sqrt{\frac{1}{2 M_{*}^{3}}} h^{M N} T_{M N}\right),
\end{aligned}
$$

where

$$
T_{M N}=2 \frac{\delta \mathcal{L}_{\Phi}}{\delta \gamma_{M N}}-\gamma_{M N} \mathcal{L}_{\Phi}
$$

Following [14], all the degrees of freedom can be disentangled using field redefinitions and Faddeev-Popov gauge fixing. Defining $\hat{h}_{M N}=(k z)^{2} h_{M N}$, then splitting the graviton components as

$$
\begin{aligned}
\tilde{h}_{\mu \nu} & =\hat{h}_{\mu \nu}-\frac{1}{4} \eta_{\mu \nu} h_{\rho}^{\rho}, & B_{\mu} & =\frac{\sqrt{2}}{k z} \hat{h}_{\mu 5}, \\
\chi & =\frac{1}{2}\left(\hat{h}_{\mu}^{\mu}-2 \hat{h}_{55}\right), & \phi & =\frac{\sqrt{3}}{\sqrt{2}(k z)^{2}} \hat{h}_{55},
\end{aligned}
$$

and defining the sources

$$
\tilde{T}_{\mu \nu}=T_{\mu \nu}-\frac{1}{4} \eta_{\mu \nu} T_{\rho}^{\rho}, \quad \tilde{T}_{55}=T_{55}+\frac{1}{2} T_{\rho}^{\rho},
$$

the graviton action $S^{h}=\int d x^{M} \mathcal{L}^{h}$ takes the simple form

$$
\begin{aligned}
\mathcal{L}^{h}= & \frac{1}{2}\left(\frac{1}{(k z)^{3}}\left(\partial_{R} \tilde{h}_{\mu \nu}\right)^{2}+\frac{1}{k z}\left(\partial_{R} B_{\mu}\right)^{2}\right. \\
& \left.+k z\left(\partial_{R} \phi\right)^{2}-\frac{1}{(k z)^{3}}\left(\partial_{R} \chi\right)^{2}\right) \\
& +\frac{1}{\sqrt{M_{*}^{3}}}\left(\frac{1}{\sqrt{2}(k z)^{3}} \tilde{h}^{\mu \nu} \tilde{T}_{\mu \nu}+\frac{1}{(k z)^{2}} B^{\mu} T_{\mu 5}\right. \\
& \left.+\frac{1}{2 \sqrt{2}(k z)^{3}} \chi T_{\mu}^{\mu}+\frac{1}{\sqrt{3} k z} \phi \tilde{T}_{55}\right) .
\end{aligned}
$$

In Eq. (53), all contractions are done with the Minkowski metric. The zero mode of $\tilde{h}_{\mu \nu}$ matches the $4 \mathrm{D}$ graviton in the 4D low-energy EFT. The zero mode of $\phi$ corresponds to the radion field. As expected, the $\chi$ field has "wrong-sign" kinetic term and is an unphysical degree of freedom.

The Feynman propagators in position-momentum space for the degrees of freedom $\left(\tilde{h}_{\mu \nu}, B_{\mu}, \phi, \chi\right)$ are expressed in terms of the scalar propagator by

$$
\begin{gathered}
\left\langle\tilde{h}_{\mu \nu}(p, z) \tilde{h}_{\rho \sigma}\left(-p, z^{\prime}\right)\right\rangle=\left.\eta_{\mu \nu} \eta_{\rho \sigma} \Delta_{p}\left(z, z^{\prime}\right)\right|_{\alpha=2, b_{i}=0}, \\
\left\langle B_{\mu}(p, z) B_{\nu}\left(-p, z^{\prime}\right)\right\rangle=\left.\eta_{\mu \nu} \Delta_{p}\left(z, z^{\prime}\right)\right|_{\alpha=1, b_{i}=\infty} \\
\left\langle\phi(p, z) \phi\left(-p, z^{\prime}\right)\right\rangle=\left.\Delta_{p}\right|_{\alpha=0, b_{i}=0}
\end{gathered}
$$




$$
\left\langle\chi(p, z) \chi\left(-p, z^{\prime}\right)\right\rangle=-\left.\Delta_{p}\right|_{\alpha=2, b_{i}=0} .
$$

The value $b_{i}=\infty$ means that boundary conditions are Dirichlet for the vector component.

The stress-energy tensor in our study is the one of the bulk scalar $\Phi$,

$$
T_{M N}=\partial_{M} \Phi \partial_{N} \Phi-\frac{1}{2} \gamma_{M N}\left(\partial_{M} \Phi \partial^{M} \Phi-M_{\phi}^{2} \Phi^{2}\right) .
$$

The sources for the disentangled graviton components are thus

$$
\begin{aligned}
\tilde{T}_{\mu \nu} & =\partial_{\mu} \Phi \partial_{\nu} \Phi-\frac{1}{4} \eta_{\mu \nu}\left(\partial_{\mu} \Phi\right)^{2} \\
T_{\mu}^{\mu} & =-\left(\partial_{\mu} \Phi\right)^{2}+2\left(\partial_{5} \Phi\right)^{2}+\frac{2}{(k z)^{2}} M^{2} \Phi^{2} \\
\tilde{T}_{55} & =\frac{3}{2}\left(\partial_{5} \Phi\right)^{2}+\frac{1}{2(k z)^{2}} M^{2} \Phi^{2} \\
T_{\mu 5} & =\partial_{\mu} \Phi \partial_{5} \Phi
\end{aligned}
$$

Having all the Feynman rules needed, we can now calculate the imaginary part of the self-energy

$$
\begin{aligned}
& \operatorname{Im}_{p}\left(z, z^{\prime}\right)=\operatorname{Im} \Pi_{p}^{h}\left(z, z^{\prime}\right)+\operatorname{Im} \Pi_{p}^{B}\left(z, z^{\prime}\right) \\
& +\operatorname{Im} \Pi_{p}^{\phi}\left(z, z^{\prime}\right)+\operatorname{Im} \Pi_{p}^{\chi}\left(z, z^{\prime}\right),
\end{aligned}
$$

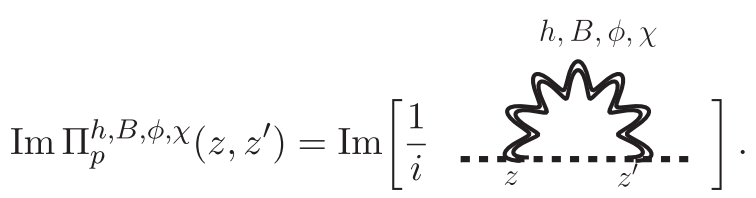

Our calculation being in position-momentum space, the vertices from the stress-energy tensor contain $\partial_{5}$ derivatives acting on the external $\Phi$ propagators. For our purposes, it is convenient to integrate by part in $z$ for each insertion of $\Pi_{p}\left(z, z^{\prime}\right)$ in the geometric series, such that these $\partial_{5}$ derivatives act instead on all propagators and metric factors inside the loop. As in previous section, we focus for simplicity on end points lying away for the UV brane. Hence, the UV brane terms and the brane contributions generated when integrating by part are irrelevant. Elements of the graviton loop calculation are given in Appendix B.

Introducing the 5D gravity coupling strength $\kappa \equiv$ $\sqrt{k^{3} / M_{*}^{3}}=k / M_{\mathrm{Pl}}$, the final result is found to be

$$
\begin{aligned}
& \operatorname{Im} \Pi^{h}\left(z, z^{\prime}\right)+\operatorname{Im} \Pi^{\chi}\left(z, z^{\prime}\right) \\
& \quad=\frac{\kappa^{2} k p^{2}}{64 \pi} \frac{2+3 \alpha}{\Gamma(1+\alpha) \Gamma(2+\alpha)} \frac{1}{\left(k z_{>}\right)^{8}}\left(\frac{z_{<}}{4 z_{>}}\right)^{\alpha-1},
\end{aligned}
$$

$\operatorname{Im} \Pi^{B}\left(z, z^{\prime}\right)=\frac{\kappa^{2} k p^{2}}{32 \pi} \frac{(2+\alpha)(3+2 \alpha)}{\Gamma(1+\alpha) \Gamma(2+\alpha)} \frac{1}{\left(k z_{>}\right)^{6}}\left(\frac{z_{<}}{4 z_{>}}\right)^{\alpha+1}$,

$\operatorname{Im} \Pi^{\phi}\left(z, z^{\prime}\right)=\frac{\kappa^{2} k^{3}}{192 \pi} \frac{(2+\alpha)\left(2+6 \alpha+\alpha^{2}\right)}{\Gamma^{2}(1+\alpha)} \frac{1}{\left(k z_{>}\right)^{6}}\left(\frac{z_{<}}{4 z_{>}}\right)^{\alpha-1}$.

It turns out that the $h, \chi, B$ contributions are small with respect to the contributions from $\phi$. They are both suppressed by a factor of $p^{2} / k^{2}$, and the $h+\chi$ contribution has also an extra suppression in $1 /\left(k z_{>}\right)^{2}$. In contrast, the contribution from the $\phi$ degree of freedom is similar to the scalar case described in the previous section and can be treated the same way.

Assuming $\alpha>1$, one can use the position-space narrow width approximation

$$
\begin{aligned}
\Pi^{\phi}\left(z, z^{\prime}\right)= & \delta\left(z-z^{\prime}\right) F_{0}^{\phi}(z)+F_{1}^{\phi}(z) \delta^{\prime}\left(z-z^{\prime}\right) \\
& +\frac{F_{2}^{\phi}(z)}{2} \delta^{\prime \prime}\left(z-z^{\prime}\right)+\cdots \\
F_{0}^{\phi}(z)= & \kappa^{2} k^{2} C_{0}^{\phi} \frac{1}{(k z)^{5}}, \quad F_{1}^{\phi}(z)=\kappa^{2} k C_{1}^{\phi} \frac{1}{(k z)^{4}}, \\
F_{2}^{\phi}(z)= & \kappa^{2} C_{2}^{\phi} \frac{1}{(k z)^{3}},
\end{aligned}
$$

with

$$
\begin{aligned}
\left(\begin{array}{c}
C_{0}^{\phi} \\
C_{1}^{\phi} \\
C_{2}^{\phi}
\end{array}\right)= & \frac{1}{48 \pi} \frac{(2+\alpha)\left(2+6 \alpha+\alpha^{2}\right)}{4^{\alpha} \Gamma^{2}(1+\alpha)} \\
& \times\left(\begin{array}{c}
\frac{1}{\alpha}+\frac{1}{\alpha+4} \\
\frac{1}{\alpha+1}+\frac{1}{\alpha+3}-\frac{1}{\alpha}-\frac{1}{\alpha+4} \\
\frac{2}{\alpha+2}+\frac{1}{\alpha}+\frac{1}{\alpha+4}-\frac{2}{\alpha+1}-\frac{2}{\alpha+3}
\end{array}\right) .
\end{aligned}
$$

Then, as in the previous section, the $C_{2}^{\phi}$ term turns out to induce an exponential suppression of the propagator in the IR region

$$
\Delta_{p}\left(z, z^{\prime}\right) \propto e^{-\kappa^{2} C_{2}^{\phi} / 4 p z_{>}} .
$$

Having an estimate at strong coupling is instructive. In the case of AdS gravity, strong coupling is at $\kappa=O(1)$ 
[13]. Using $\kappa=1$, a point with self-consistent truncation of the loop (see previous section) is, for instance,

$$
\kappa=1, \quad \alpha=2, \quad z_{>} \lesssim \frac{2.6}{p} .
$$

Again, as in the scalar cubic interaction case, the truncation lies at the limit of validity of region 2 . Weaker values of $\kappa$ or higher $\alpha$ would require to know the contributions from regions 3 to 4 to get an accurate result; however, this is beyond the scope of the present calculations.

\section{EFT CONSIDERATIONS}

\section{A. Censorship of the IR region}

In the two previous sections, we have found that, at least at strong coupling and for $O(1)$ values of the bulk mass parameter [see the examples Eqs. (47) and (72)], the opaque region of position-momentum space starts around $p z_{>}=O(1)$. Let us compare this result to estimates of the region where the 5D EFT breaks down. Following the approach of [6], one includes a higher dimension bilinear operator in the effective 5D Lagrangian

$$
\mathcal{L}_{5} \supset \frac{1}{\Lambda^{2}} \square^{2} \Phi^{2}
$$

This operator is certainly expected to dominate the propagator in some region of position-momentum space, signaling that the 5D EFT breaks down. Dressing the 5D propagator with the insertion from Eq. (73), we can readily observe that in the $p>1 / z_{>}$region (i.e. region 3 ), the $k z \partial_{5}$ from the extra derivatives contributes to extra $\frac{k}{\Lambda} p z_{>}$factors in the geometric series resulting from the dressing. From this, one concludes that the 5D EFT becomes invalid around

$$
\frac{k}{\Lambda} p z_{>} \sim 1
$$

A similar result was obtained in [6] by projecting the propagator onto a single KK mode.

An estimate of $\Lambda$ is given by dimensional analysis $[12,13]$

$$
\Lambda^{3} \sim 24 \pi^{3} M_{*}^{3}=24 \pi^{3} \frac{k^{3}}{\kappa^{2}} .
$$

For strongly coupled gravity $\kappa \sim 1$, the region of EFT breaking starts thus at $p \sim 10 / z_{>}$. This is typically inside the "opaque" region where exponential suppression occurs. Hence, there is no need to forbid by hand the region of 5D EFT breaking, the theory censors this region by itself. For weaker couplings, our estimates are not precise enough to draw a similar conclusion, because contributions from regions 3 to 4 should also be taken into account in the dressing (see Sec. V).

It is worth noting that the $\kappa$-dependence of the EFT breaking region Eq. (74) obtained from a naive dimensional analysis Eq. (75) does not match the $\kappa$-dependence of the opaque region induced by gravitational dressing. The former goes as $p z_{>} \propto 1 / \kappa^{2 / 3}$, while the latter goes as $p z_{>} \propto 1 / \kappa^{2}$. Interestingly, this slightly disturbing feature can be avoided if one takes the hypothesis that the higher dimensional operators in the theory are only generated from gravity loops. Indeed, the bilinear higher derivative operators of the kind of Eq. (73) are then generated by contributions from gravitational dressing, i.e. from the loop diagram shown in Eq. (64). In such case, the boundary of the EFT breaking region scales as $p z_{>} \propto 1 / \kappa^{2}$, just like the opaque region. In fact, the real part of $\Pi_{p}$ induces effective operators which are automatically of same order of magnitude as the imaginary part of $\Pi_{p}$. As a result, the 5D EFT breaking region and the opacity region should roughly match each other for any values of coupling and bulk mass, since the two effects originate from the same loop. Somehow, the dressing by $\operatorname{Im}_{p}$ renders automatically opaque, the region where the effects from $\operatorname{Re} \Pi_{p}$ would be out of control.

Finally, let us emphasize again that the IR opacity and censorship behaviors discussed in this work have no equivalent in flat space. First, unlike in the curved case, it is possible to have exact KK parity in a flat extradimension. In such case, the KK modes are stable, hence the $\Pi_{p}\left(z, z^{\prime}\right)$ self-energy does not develop an imaginary part and no effect of opacity can ever occur. Second, even if KK parity is broken, the KK modes couple to bulk fields with the strength of $4 \mathrm{D}$ gravity, hence very weakly. The dressing of a bulk field by KK modes becomes relevant only near the $5 \mathrm{D}$ cutoff, once enough real KK modes run in the loops, and thus does not seem to have interesting consequences.

\section{B. CFT interpretation}

The interpretation of the opacity effect in a dual CFT picture is clear and was already noticed in [9] in the spacelike momentum case.

First, let us remind that the presence of the IR brane is interpreted in the CFT dual as a spontaneous breaking of conformal symmetry in the IR, and any field localized toward the IR brane is interpreted as a bound state arising from confinement of the CFT (see, e.g., [17]). Concretely, the UV-to-UV correlators, which are those needed for holography, interact and possibly mix with the IR-localized fields. At low four-momentum $|p| \sim \mu$, most KK modes are integrated out, and only light poles-including those from the IR-localized fields-are in the spectrum, and are understood as the resonances arising in the confined phase of the CFT. In contrast, at momentum $|p| \gg \mu$, IR opacity implies that the effects from IR-brane-localized degrees of 
freedom (e.g., poles) vanish exponentially from the UV-to-UV correlators. In fact, the IR brane vanishes from the correlators, which become effectively the same as in pure AdS.

On the CFT side, the vanishing of the IR-related effects in the correlators matches the intuition about bound states of size $\sim 1 / \mu$. At momentum above $\mu$, an external probe sees the CFT constituents and does not know about the bound states. The low-energy EFT describing the bound states breaks at a scale somewhat above $\mu$ (say $4 \pi \mu$ ); however, these vanish from the amplitudes via the effect of form factors-which are implemented by the opacity property of AdS. Such observations were essentially done in [9] for spacelike four-momentum, and the present work ensures they apply also for timelike four-momentum.

The above considerations on the low-energy EFT of "CFT bound states" in the IR involve the absolute fourmomentum $p$ and how it compares to the "CFT breaking scale" $\mu$. The meaning of $p^{\mu}$ is trivial on both CFT and AdS sides. It is worth pointing out that another set of conclusions, with apparently no or little interplay with the aspect discussed above, is reached when considering the 5D short distance limit of small $\left|\Delta X^{M}\right| \sim 1 / \Lambda$. In this limit, curvature becomes negligible, and the same cutoff as in $5 \mathrm{D}$ flat space is reached. The existence of the 5D cutoff, while conceptually simple on the AdS side, has nontrivial implications for the CFT side. A simple example is as follows. A 5D mass cannot be larger than $\Lambda$. On the CFT side, this implies that the corresponding conformal dimension cannot be arbitrary large. Hence, validity of the 5D EFT implies a nontrivial bound on the dimension of the CFT operator. Such constraint on the CFT side is, to the best of our understanding, unrelated to the CFT's IR behavior discussed above. Here the parameter involved to delineate the limit of the 5D EFT is the 5D energy, whose CFT interpretation is not obvious and has to be carefully determined. An analysis along these lines involves identifying the AdS Hamiltonian with the CFT dilatation operator. These aspects have been thoroughly studied in [18] from both AdS and CFT sides.

\section{KK CONTINUUM CASCADE DECAY RATE}

In the previous sections, we have found that opacity of Lorentzian AdS is a consequence of bulk interactions and happens essentially because KK modes can decay into lighter KK modes. The KK modes can often have a width larger than $\mu$ in which case they tend to form a continuum. The term "KK modes" is general since it applies to a set of modes with any width, but in the following we will use specifically "KK continuum" for a set of dressed KK modes in internal lines. The AdS KK continuum can undergo cascade decays, and there are various reasons to think about them in detail.

First, one may wonder whether the IR brane actually remains invisible to a bulk field with $p \gg \mu$ in the presence of cascade decays. Indeed, consider, for instance, a process where a KK continuum is created on the UV brane, propagates to the IR brane where it decays into IR localized states. As we have seen in previous sections, for $p>\mu$, this process is exponentially suppressed. However, the $\mathrm{KK}$ continuum could also decay in the bulk, splitting its initial timelike absolute momentum $p$ between the various daughter continuums produced. Repeating the decay process enough times, the absolute momentum keeps fragmenting and the final daughter KK modes end up with a four-momentum $p_{f} \sim O(\mu)$. Naively this seems to imply that the probability of the initial continuum to reach the IR brane is not exponentially suppressed. This observation seems to somewhat contradict the picture of IR opacity we have previously obtained, and therefore some investigation is needed.

Second, the cascade decay of the KK continuum is certainly interesting in its own right and especially in the context of the AdS/CFT correspondence. Cascade decays in the dual theory correspond to the decay of excitations in the CFT, which are expected to produce soft, spherically distributed final states with high multiplicity, sometimes called "soft bombs"; see, e.g., [19]. While qualitative studies have been done on the CFT side, not so many studies have been on the AdS side, to the best of our knowledge. A Monte Carlo study has been done in [20] in the KK picture, considering the decay of single KK modes, with a focus on the details of the final state distribution. In our analytical calculation, we will recover qualitatively some of these features, but our focus will rather be on the estimation of the total rate of the cascade decay. It is not clear if a same calculation has been tried on the CFT side.

For simplicity, we focus on a scalar field decaying via a cubic self-interaction, which is enough to display the key features of the calculation. The interaction Lagrangian is $\mathcal{L} \supset \lambda \Phi^{3}$. Other interactions like gravity are assumed to be present such that the exponential suppression starts around $p \sim 1 / z_{>}$, which will be used to truncate the space integrals.

Our main goal is to estimate the rate of a cascade decay. Importantly, since the KK modes tend to be broad, a 4D narrow-width approximation cannot be applied. This means that the rate cannot be factored out as production rate times many branching ratios, as would be the case for the cascade decay of narrow particles. Instead the whole squared matrix element has to be calculated without relying on a narrow width approximation.

For our purposes it is enough to study the rate of a threebody cascade decay, made of two successive decays induced by the cubic vertex. In terms of KK modes, the integrated square matrix element is given by

$$
\sum_{m, n, p} \int d \Phi_{3}\left|\mathcal{M}_{m n p}\right|^{2}
$$


with

$$
\mathcal{M}_{m, n, p}=\underbrace{p}_{(p)} \overbrace{(m)}^{(n)}=\int_{\frac{1}{k}}^{\frac{1}{\mu}} d u \int_{\frac{1}{k}}^{\frac{1}{\mu}} d v \frac{\lambda^{2}}{(k u)^{5}(k v)^{5}} \Delta_{p}\left(z_{0}, u\right) f_{p}(u) \Delta_{q}(u, v) f_{m}(v) f_{n}(v) \text {. }
$$

The exponential falloff of the propagators resulting from dressing - in particular gravitational dressing - is assumed to occur around $p \sim 1 / z_{>}$. The suppression of the propagator amounts to cut the $u, v$ position integrals. Notice that the EFT of the external KK modes breaks down in the IR region, for instance, for $v \gg 1 / q$ in Eq. (77), but as discussed in Sec. VII A this region is censored by the exponential falloff of the propagators to which the external KK modes are connected.

Let us write the three-body phase space integral as the sequence of two two-body decays

$$
\begin{aligned}
& \int d \Phi_{3}\left(p ; p_{1}, p_{2}, p_{3}\right) \\
& =\int \frac{d q^{2}}{2 \pi} \int d \Phi_{2}\left(p ; q, p_{3}\right) \int d \Phi_{2}\left(q ; p_{1}, p_{2}\right)
\end{aligned}
$$

and focus on the secondary decay. In Eq. (76), the expression describing this subdecay is

$$
\begin{aligned}
& \sum_{m, n} \int_{\frac{1}{k}}^{\frac{1}{\mu}} d v \int_{\frac{1}{k}}^{\frac{1}{\mu}} d v^{\prime} \int d \Phi_{2}\left(q ; p_{1}, p_{2}\right) \frac{\lambda^{2}}{(k v)^{5}\left(k v^{\prime}\right)^{5}} \\
& \quad \times \Delta_{q}(u, v) \Delta_{q}^{*}\left(u^{\prime}, v^{\prime}\right) f_{m}(v) f_{m}\left(v^{\prime}\right) f_{n}(v) f_{n}\left(v^{\prime}\right) .
\end{aligned}
$$

Let us first use the contour integral trick on the KK sums and evaluate the phase space integral. The expression becomes

$$
\begin{aligned}
& \frac{1}{64 \pi^{3}} \int_{\frac{1}{k}}^{\frac{1}{\mu}} d v \int_{\frac{1}{k}}^{\frac{1}{\mu}} d v^{\prime} \frac{\lambda^{2}}{(k v)^{5}\left(k v^{\prime}\right)^{5}} \Delta_{q}(u, v) \Delta_{q}^{*}\left(u^{\prime}, v^{\prime}\right) \\
& \quad \times \oint_{\mathcal{C}[\sqrt{\rho}]} d \rho \oint_{\mathcal{C}[q-\sqrt{\rho}]} d \tilde{\rho} K(q ; \sqrt{\rho}, \sqrt{\tilde{\rho}}) \Delta_{\sqrt{\rho}}\left(v, v^{\prime}\right) \Delta_{\sqrt{\tilde{\rho}}}\left(v, v^{\prime}\right) .
\end{aligned}
$$

Up to this point these are exact expressions; now let us make approximations. Since the internal propagators are exponentially suppressed because of their dressing, the integration in position-momentum space is restricted to $u, u^{\prime}, v, v^{\prime} \lesssim 1 / q$, i.e. the internal propagators are taken to be nonvanishing only in region 2 . It follows that, because of the kinematic threshold, the $\Delta_{\sqrt{\rho}}$ 's appearing in the final state contour integration are also forced to be in region 2 since $\sqrt{\rho} \leq q$. Apart from the restriction of the integration region, the other important approximation we take is to replace the kinematic factor as $K(q ; \sqrt{\rho}, \sqrt{\tilde{\rho}}) \approx$ $\Theta(q-\sqrt{\rho} / 2) \Theta(q-\sqrt{\tilde{\rho}} / 2)$. This is a convenient but rough approximation implying extra $O(1)$ uncertainties in the subsequent results. Such level of precision is sufficient for our study.

Given the approximations above, the expression becomes

$$
\begin{aligned}
& \frac{1}{64 \pi^{3}} \int_{\frac{1}{k}}^{\frac{1}{q}} d v \int_{\frac{1}{k}}^{\frac{1}{q}} d v^{\prime} \frac{\lambda^{2}}{(k v)^{5}\left(k v^{\prime}\right)^{5}} \Delta_{q}(u, v) \Delta_{q}^{*}\left(u^{\prime}, v^{\prime}\right) \\
& \quad \times \oint_{\mathcal{C}[q / 2]} d \rho \oint_{\mathcal{C}[q / 2]} d \tilde{\rho} X_{\sqrt{\rho}}\left(v, v^{\prime}\right) X_{\sqrt{\tilde{\rho}}}\left(v, v^{\prime}\right),
\end{aligned}
$$

where we have introduced $X_{q}\left(z, z^{\prime}\right)$, the nonanalytic part of $\Delta_{q}\left(z, z^{\prime}\right)$ in region 2 . We then integrate over the positions $v$, $v^{\prime}$. The integration is straightforward but slightly tedious because, as discussed in Sec. III, $\Delta_{q}$ in region 2 contains several terms. It turns out that the nonanalytic term $X_{q}$ dominates because it has the largest positive power of $v$ 's. The result reads

$$
\frac{\Gamma^{2}(-\alpha)}{4096 \pi 4^{6 \alpha}(3 \alpha+2)^{2} \Gamma^{4}(\alpha+1) \Gamma^{2}(\alpha+2)} u u^{\prime}\left(q^{2} u u^{\prime}\right)^{\alpha},
$$

while the neglected terms are smaller by at least a factor $(|q| / k)$. Contributions from UV brane terms are also negligible. We then observe that Eq. (82) can be re-expressed as a function of $X_{q}\left(u, u^{\prime}\right)$,

$$
i \frac{1}{k} \frac{(-1)^{-\alpha} \Gamma(-\alpha)}{2048 \pi 4^{5 \alpha}(3 \alpha+2)^{2} \Gamma^{3}(\alpha+1) \Gamma^{2}(\alpha+2)} X_{q}\left(u, u^{\prime}\right) .
$$

Having reduced the expression of the secondary decay, let us return to the full amplitude Eq. (76). Recall we are using the phase space representation of Eq. (78). We can readily use the two-body phase space approximation on the primary decay, just as done on the secondary decay. Then let us consider the remaining integral on the $q^{2}$ variable from Eq. (78). The integrand depends on $q^{2}$ via Eq. (83). The integral is along the real axis but is related to the one over a circle by $\int_{0}^{R} d q^{2} X_{q}\left(u, u^{\prime}\right)=\int_{\mathcal{C}[R]} d q^{2} X_{q}\left(u, u^{\prime}\right) \frac{(-1)^{\alpha}}{2 i \sin (\pi \alpha)}$, 
where $R$ is taken at $p / 2$ since one uses the same approximations as in the secondary decay. As defined in Sec. IV, the contour is $\left.\left.\mathcal{C}[R]=\left(R e^{i \theta} \mid \theta \in\right]-2 \pi, 0\right]\right)$. With these rewritings, the $q^{2}$ integral of $X_{q}$ has the same form as the contour integral representing a sum of KK modes in final state. This means that we have reduced the integrated three-body square amplitude into an integrated two-body square amplitude. Putting all the pieces together, the relation found is

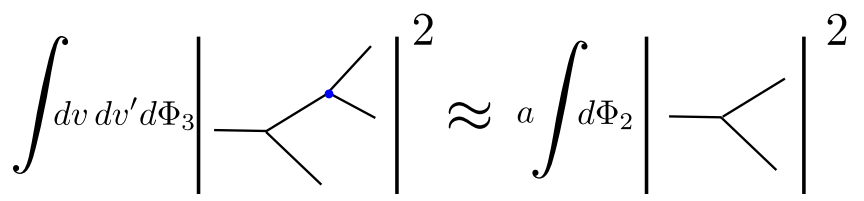

where

$a=\frac{\lambda^{2}}{k} \frac{1}{4096 \pi^{2} 4^{5 \alpha}(3 \alpha+2)^{2}} \frac{\Gamma^{2}(-\alpha)}{\Gamma^{2}(\alpha+1) \Gamma^{2}(\alpha+2)}$.

The approximate recursion relation Eq. (84) can be applied to a cascade decay with any number of final states. It provides therefore a very simple way to estimate the rate of a cascade decay: a cascade decay with $n$ branchings carries a weight $a^{n}$. Importantly, $a$ turns out to be much smaller than 1 . Even if one takes $\lambda$ to a large value such that the exponential suppression comes only from scalar dressing, using for example the strong coupling point Eq. (47), the $a$ coefficient still remains suppressed by 2 orders of magnitude. The calculation done here is only for a scalar interaction; however, similar qualitative properties can be expected for gravitons.

Having a way to estimate cascade decay rates, let us consider qualitatively a typical cascade decay. The propagators in the cascade decay diagram live in region 2 and are thus proportional to $p^{2 \alpha}$. This implies that large values of $p^{2}$ are preferred in the kinematic integrals. This favors large energy and small three-momenta in the outgoing legs. One thus recognizes the familiar tendency of the KK modes to decay into near-threshold states-a result expected from approximate 5D Lorentz invariance and already studied in [20]. Since the boost with respect to the daughter particle is small, spatial correlations between final states are small and the cascade decays of a bulk field leads to spherical events. Still because of the $p^{2 \alpha}$ factors, a decay $a \rightarrow 1+2$ tends to prefer equal outgoing $p_{\text {daughters }} \approx \frac{p}{2}$. These features provide a qualitative picture of the most favored kinematic configuration: each KK continuum splits into two KK continuum sharing half of the absolute four-momentum. ${ }^{13}$

We can use this typical kinematic configuration to get an idea of the rate as a function of the typical final state

\footnotetext{
${ }^{13}$ This is somehow the continuous version of a KK mode with mass $m_{n}$ decaying into two modes with mass $m_{\text {daughters }} \approx m_{n} / 2$.
}

momentum. The initial field is assumed to have absolute timelike momentum $p$. The decay ends at a typical absolute momentum $p_{f}$ which could for instance be the typical momentum for which the KK modes are long lived enough to escape the detector. Assuming that the absolute momentum is divided by 2 at each branching, we have $p / p_{f} \sim 2^{n}$. Consider that such splittings occur $n$ times, such that the full cascade decay diagram has $2^{n}-1$ vertices. The square amplitude is proportional to $a^{2^{n}-1}$. As a result, the square amplitude for this cascade decay diagram is suppressed by

$$
\Gamma \sim a^{p / p_{f}-1} .
$$

Since $a$ is much smaller than 1 , this continuum decay rate is exponentially suppressed as a function of the initial momentum.

This last part of the analysis is of course very qualitative but it applies to the most likely kinematic configuration; hence, it is a good hint that bulk cascade decays are exponentially suppressed as a function of the momentum of the initial continuum. Numerical evaluations, perhaps using Monte Carlo integration, could be used to test this claim. It would also be interesting to evaluate the "soft bomb" rate directly from the CFT side.

\section{CONCLUSION}

In this note, we have shown that propagators in a truncated Lorentzian $\mathrm{AdS}_{5}$ background are exponentially suppressed in the IR region of the bulk when the conformal coordinate $z$ exceeds a threshold of $O(1 /|p|)$, with $p=\sqrt{p^{\mu} p_{\mu}}$. While such an "opacity" property is trivial for spacelike momentum, for timelike momentum we show that it is a consequence of the dressing of 5D propagators by bulk interactions. AdS gravity induces a universal contribution to the dressing, and we find that the leading effect comes from the scalar component of the 5D graviton.

Exact loop calculations in AdS are notoriously difficult, and the calculations in this work rely instead on a set of approximations, the main ones being (i) the limits of the AdS propagator in different regions of position-momentum space and (ii) a 5D position-momentum space narrowwidth expansion. Part of the results has been checked numerically using exact expressions. Our results tend to be more exact at strong coupling, while for weaker coupling extra contributions to the dressing from regions 3 to 4 may also matter, such that the opacity effect obtained here may be underestimated.

We find that, at least at strong coupling, the exponential suppression "censors" the region of position-momentum space where the 5D EFT would become invalid. We argue that under certain conditions this effect may be valid for any strength of 5D gravity. The properties of opacity and censorship discussed in this work are inherent to curved space and do not occur in flat space. 
Building on these results we turn to another feature of Lorentzian AdS: the cascade decay of bulk fields. These are known to give soft spherical events with high multiplicity, a property also expected from large- $N$ strongly interacting CFT sectors. Focusing on a cubic interaction, using the techniques previously developed and a rough approximation on threshold effects, we obtain an approximate recursion relation between cascade decay rates with $n$ and $n+1$ final states. Qualitative considerations then suggest that the rate for bulk cascade decays is exponentially suppressed as a function of the momentum $p$ of the initial state. It would be interesting to pursue such study with more accurate calculations or perhaps numerical tools, both on the AdS and CFT sides.

The set of results obtained in this note can be seen as a contribution to establishing a solid understanding of effective field theories in AdS. But one may also find it inspiring in the scope of physics beyond the Standard Model, as it makes clear that the truncated AdS background can be used to describe a strongly interacting dark sector which somehow vanishes at high energy. This direction will be pursued in the companion paper [7].

\section{ACKNOWLEDGMENTS}

I am grateful to Flip Tanedo, Csaba Csáki, David Poland, Eric Perlmutter, Mark Wise, Prashant Saraswat, Gero von Gersdorff, Eduardo Pontón, and Philippe Brax for useful discussions and comments. I thank UCR where part of this work was realized. This work is supported by the São Paulo Research Foundation (FAPESP) under Grants No. 2011/ 11973, No. 2014/21477-2, and No. 2018/11721-4.

\section{APPENDIX A: DRESSED PROPAGATOR IN KK REPRESENTATION}

For completeness, we show how the dressed KK representation of $\Delta_{q}\left(z, z^{\prime}\right)$ follows from the general dressed equation of motion Eq. (31). This is somewhat standard but will make clear that the KK modes get generally mixed by their self-energies.

Let us introduce the matrix notation

$$
\mathbf{f}(z)=\left(f_{n}(z)\right) \mathbf{D}=\left(\frac{\delta_{n p}}{q^{2}-m_{n}^{2}}\right)
$$

where $\mathbf{f}$ is a one-dimensional infinite vector and $\mathbf{D}$ is an infinite diagonal matrix.

The free propagator reads

$$
\Delta_{q}^{(0)}\left(z, z^{\prime}\right)=i \mathbf{f}(z) \cdot \mathbf{D} \cdot \mathbf{f}\left(z^{\prime}\right) .
$$

Starting from the geometric series representation of the solution to Eq. (31),

$$
\begin{aligned}
\Delta_{p}\left(z, z^{\prime}\right)= & \Delta_{q}^{(0)}\left(z, z^{\prime}\right) \\
& +\int d u \int d v \Delta_{q}^{(0)}(z, u) i \Pi(u, v) \Delta_{q}^{(0)}\left(v, z^{\prime}\right)+\ldots \\
= & \Delta_{q}^{(0)}\left(z, z^{\prime}\right)+\int d u \int d v \mathbf{f}(z) \cdot i \mathbf{D} \cdot \mathbf{f}(u) i \Pi(u, v) \\
& \times \mathbf{f}(v) \cdot i \mathbf{D} \cdot \mathbf{f}\left(z^{\prime}\right)+\cdots,
\end{aligned}
$$

we see that the matrix

$$
\begin{aligned}
i \Pi & \equiv \int d u \int d v i \Pi(u, v) \mathbf{f}(u) \otimes \mathbf{f}(v) \\
& =\left(\int d u \int d v i \Pi(u, v) f_{n}(u) f_{m}(v)\right)
\end{aligned}
$$

appears. This is the self-energy matrix for the entire set of KK modes and it is in general nondiagonal. Keeping only the imaginary part of the self-energy and summing the series gives the dressed KK representation

$$
\Delta_{q}\left(z, z^{\prime}\right)=i \mathbf{f}(z) \cdot\left[\mathbf{D}^{-1}+i \operatorname{Im} \Pi\right]^{-1} \cdot \mathbf{f}\left(z^{\prime}\right),
$$

where []$^{-1}$ is the matrix inverse. This makes clear that the full nondiagonal self-energy matrix has to be in principle included in the propagator, which nontrivially mixes the KK modes.

\section{APPENDIX B: GRAVITON LOOP}

Let us introduce the notations

$$
\begin{aligned}
& \Delta_{p}^{h}\left(z, z^{\prime}\right)=\left.\Delta_{p}\left(z, z^{\prime}\right)\right|_{\alpha=2, b_{i}=0}, \\
& \Delta_{p}^{B}\left(z, z^{\prime}\right)=\left.\Delta_{p}\left(z, z^{\prime}\right)\right|_{\alpha=1, b_{i}=\infty}, \\
& \Delta_{p}^{\phi}\left(z, z^{\prime}\right)=\left.\Delta_{p}\right|_{\alpha=0, b_{i}=0}, \\
& \Delta_{p}^{\chi}\left(z, z^{\prime}\right)=-\left.\Delta_{p}\right|_{\alpha=2, b_{i}=0} .
\end{aligned}
$$

The propagators of the graviton degrees of freedom in region 2 and away from the poles are given by

$\Delta^{h}\left(z, z^{\prime}\right)=i \frac{2 k}{p^{2}}+i \frac{2 \gamma-1+2 \log (p / 2 k)-\pi \tan (p / \mu+\pi / 4)}{2 k}$,

$\Delta^{\chi}\left(z, z^{\prime}\right)=-i \frac{2 k}{p^{2}}-i \frac{2 \gamma-1+2 \log (p / 2 k)-\pi \tan (p / \mu+\pi / 4)}{2 k}$,

$\Delta^{\phi}\left(z, z^{\prime}\right)=i \frac{2 \gamma+2 \log (p / 2 k)-\pi \tan (p / \mu+\pi / 4)}{2 k}$ 


$$
\begin{aligned}
\Delta^{B}\left(z, z^{\prime}\right)= & i\left(\frac{1}{2 k}-\frac{k z_{<}^{2}}{2}\right)-i \frac{p^{2}\left(\left(k z_{<}\right)^{2}-1\right)}{8 k^{3}} \\
& \times(2 \gamma-1+2 \log (p / 2 k)-\pi \tan (p / \mu+\pi / 4)) .
\end{aligned}
$$

The contour integrals in region 2 over a circle of radius $1 / z_{>}$are given by

$$
\begin{aligned}
& \frac{1}{-2 \pi} \int_{\mathcal{C}\left[z_{>}^{-1}\right]} d \rho \Delta^{h}\left(\sqrt{\rho} ; z, z^{\prime}\right)=\frac{1}{2 k z_{>}^{2}}, \\
& \frac{1}{-2 \pi} \int_{\mathcal{C}\left[z_{>}^{-1}\right]} d \rho \Delta^{\chi}\left(\sqrt{\rho} ; z, z^{\prime}\right)=-\frac{1}{2 k z_{>}^{2}}, \\
& \frac{1}{-2 \pi} \int_{\mathcal{C}\left[z_{>}^{-1}\right]} d \rho \Delta^{\phi}\left(\sqrt{\rho} ; z, z^{\prime}\right)=\frac{1}{2 k z_{>}^{2}}, \\
& \frac{1}{-2 \pi} \int_{\mathcal{C}\left[z_{>}^{-1}\right]} d \rho \Delta^{B}\left(\sqrt{\rho} ; z, z^{\prime}\right)=\frac{1-\left(k z_{<}\right)^{2}}{16\left(k z_{>}\right)^{3} z_{>}} .
\end{aligned}
$$

The integral of the bulk scalar away from the UV brane is given by

$$
\frac{1}{-2 \pi} \int_{\mathcal{C}\left[z_{>}^{-1}\right]} d \rho \Delta^{\Phi}\left(\sqrt{\rho} ; z, z^{\prime}\right)=\frac{2 k^{3} z z^{\prime}}{\Gamma(\beta+1) \Gamma(\beta+2)}\left(\frac{z_{<}}{4 z_{>}}\right)^{\beta+1} .
$$

When reducing the diagrams, the following integrals are needed:

$$
\begin{aligned}
\operatorname{Im}\left[\frac{1}{i} \int \frac{d^{4} q}{(2 \pi)^{2}} \frac{1}{q^{2}(p+q)^{2}}\right] & =\frac{1}{16 \pi} \\
\operatorname{Im}\left[\frac{1}{i} \int \frac{d^{4} q}{(2 \pi)^{2}} \frac{p \cdot(p+q)}{q^{2}(p+q)^{2}}\right] & =-\frac{p^{2}}{32 \pi} \\
\operatorname{Im}\left[\frac{1}{i} \int \frac{d^{4} q}{(2 \pi)^{2}} \frac{(p \cdot(p+q))^{2}}{q^{2}(p+q)^{2}}\right] & =\frac{p^{4}}{64 \pi}
\end{aligned}
$$

The amplitudes read

$$
\begin{aligned}
\operatorname{Im} \Pi^{h+\chi}\left(z, z,^{\prime}\right)= & \frac{1}{2 M^{3}(k z)^{3}\left(k z^{\prime}\right)^{3}} \frac{1}{16 \pi} \\
& \times\left[\frac{p^{2}}{2}\left(\frac{2 m^{2}}{(k z)^{2}}+\frac{2 m^{2}}{\left(k z^{\prime}\right)^{2}}+2\left(\overleftarrow{\partial_{z}} \overrightarrow{\partial_{z}}+\overleftrightarrow{\partial_{z^{\prime}}} \overrightarrow{\partial_{z^{\prime}}}\right)\right)\right. \\
& \left.+\left(\frac{2 m^{2}}{(k z)^{2}}+2 \overleftarrow{\partial_{z}} \overrightarrow{\partial_{z}}\right)\left(\frac{2 m^{2}}{\left(k z^{\prime}\right)^{2}}+2 \overleftrightarrow{\partial_{z^{\prime}}} \overrightarrow{\partial_{z^{\prime}}}\right)\right] \\
& \times \frac{1}{4 \pi^{2}} \int_{\mathcal{C}} d \rho \Delta^{\Phi}\left(\sqrt{\rho}, z, z^{\prime}\right) \int_{\tilde{\mathcal{C}}} d \tilde{\rho} \Delta^{\chi}\left(\sqrt{\tilde{\rho}}, z, z^{\prime}\right) \\
\operatorname{Im} \Pi^{B}\left(z, z,,^{\prime}\right)= & \frac{1}{M^{3}} \frac{1}{(k z)^{2}\left(k z^{\prime}\right)^{2}} \frac{1}{16 \pi} \\
& \times\left(p^{2} \overrightarrow{\partial_{z}} \frac{\partial_{z^{\prime}}}{-p^{2}}\left(\overleftarrow{\partial_{z}} \overrightarrow{\partial_{z^{\prime}}}+\overrightarrow{\partial_{z}} \overleftrightarrow{\partial_{z^{\prime}}}\right) / 2\right) \\
& \times \frac{1}{4 \pi^{2}} \int_{\mathcal{C}} d \rho \Delta^{\Phi}\left(\sqrt{\rho}, z, z^{\prime}\right) \int_{\tilde{\mathcal{C}}} d \tilde{\rho} \Delta^{B}\left(\sqrt{\tilde{\rho}}, z, z^{\prime}\right), \\
\operatorname{Im} \Pi^{\phi}\left(z, z^{\prime}\right)= & \frac{4}{3 M^{3}} \frac{1}{(k z)\left(k z^{\prime}\right)} \frac{1}{16 \pi}\left(\frac{m^{2}}{2(k z)^{2}}+\frac{3}{2} \overleftarrow{\partial_{z}} \overrightarrow{\partial_{z}}\right) \\
& \times\left(\frac{m^{2}}{2\left(k z^{\prime}\right)^{2}}+\frac{3}{2} \overleftrightarrow{\partial_{z^{\prime}}} \overrightarrow{\partial_{z^{\prime}}}\right) \\
& \times \frac{1}{4 \pi^{2}} \int_{\mathcal{C}} d \rho \Delta^{\Phi}\left(\sqrt{\rho}, z, z^{\prime}\right) \int_{\tilde{\mathcal{C}}} d \tilde{\rho} \Delta^{\phi}\left(\sqrt{\tilde{\rho}}, z, z^{\prime}\right)
\end{aligned}
$$

The $\vec{\partial}$ derivatives act on the internal $\Delta^{\Phi}\left(\sqrt{\rho}, z, z^{\prime}\right)$ only. The $\overleftarrow{\partial}$ would act on the external $\Phi$ propagator only. However, we can formally integrate by part, replacing thus the $\overleftarrow{\partial}$ derivatives by derivatives acting on the whole loop, namely on the internal $\Delta^{\Phi}\left(\sqrt{\rho}, z, z^{\prime}\right), \Delta^{h, B, \phi, \chi}\left(\sqrt{\rho}, z, z^{\prime}\right)$, and the $z$-dependent factors from the vertices. This leads to Eqs. (65)-(67) where boundary terms have been neglected.
[1] J. M. Maldacena, The large N limit of superconformal field theories and supergravity, Int. J. Theor. Phys. 38, 1113 (1999); Adv. Theor. Math. Phys. 2, 231 (1998).

[2] E. Witten, Anti-de Sitter space and holography, Adv. Theor. Math. Phys. 2, 253 (1998).

[3] O. Aharony, S. S. Gubser, J. M. Maldacena, H. Ooguri, and Y. Oz, Large $\mathrm{N}$ field theories, string theory and gravity, Phys. Rep. 323, 183 (2000).

[4] L. Randall and R. Sundrum, A Large Mass Hierarchy from a Small Extra Dimension, Phys. Rev. Lett. 83, 3370 (1999).
[5] L. Randall and M. D. Schwartz, Quantum field theory and unification in AdS5, J. High Energy Phys. 11 (2001) 003.

[6] W. D. Goldberger and I. Z. Rothstein, High-Energy Field Theory in Truncated AdS Backgrounds, Phys. Rev. Lett. 89, 131601 (2002).

[7] P. Brax, S. Fichet, and P. Tanedo, The warped dark sector, arXiv:1906.0219.

[8] P. Breitenlohner and D. Z. Freedman, Positive energy in anti-De Sitter backgrounds and gauged extended supergravity, Phys. Lett. 115B, 197 (1982). 
[9] T. Gherghetta and A. Pomarol, The Standard Model partly supersymmetric, Phys. Rev. D 67, 085018 (2003).

[10] O. Aharony, L. F. Alday, A. Bissi, and E. Perlmutter, Loops in AdS from conformal field theory, J. High Energy Phys. 07 (2017) 036.

[11] S. Giombi, C. Sleight, and M. Taronna, Spinning AdS loop diagrams: Two point functions, J. High Energy Phys. 06 (2018) 030.

[12] Z. Chacko, M. A. Luty, and E. Ponton, Massive higher dimensional gauge fields as messengers of supersymmetry breaking, J. High Energy Phys. 07 (2000) 036.

[13] K. Agashe, H. Davoudiasl, G. Perez, and A. Soni, Warped gravitons at the LHC and beyond, Phys. Rev. D 76, 036006 (2007).

[14] E. Dudas and G. von Gersdorff, Universal contributions to scalar masses from five dimensional supergravity, J. High Energy Phys. 10 (2012) 100.
[15] E. E. Boos, Y. A. Kubyshin, M. N. Smolyakov, and I. P. Volobuev, Effective Lagrangians for physical degrees of freedom in the Randall-Sundrum model, Classical Quantum Gravity 19, 4591 (2002).

[16] K. Hinterbichler, Theoretical aspects of massive gravity, Rev. Mod. Phys. 84, 671 (2012).

[17] N. Arkani-Hamed, M. Porrati, and L. Randall, Holography and phenomenology, J. High Energy Phys. 08 (2001) 017.

[18] A. L. Fitzpatrick, E. Katz, D. Poland, and D. SimmonsDuffin, Effective conformal theory and the flat-space limit of AdS, J. High Energy Phys. 07 (2011) 023.

[19] M. J. Strassler, Why unparticle models with mass gaps are examples of hidden valleys, arXiv:0801.0629.

[20] C. Csaki, M. Reece, and J. Terning, The AdS/QCD correspondence: Still undelivered, J. High Energy Phys. 05 (2009) 067. 